\title{
School dental screening does not increase dental attendance rates or reduce disease levels
}

\author{
Does school dental screening impact upon untreated dental disease or dental \\ attendance at a population level?
}

\author{
Milsom K, Blinkhorn A, Worthington H, Threlfall A, Buchanan \\ K, Kearney-Mitchell P, Tickle M. The effectiveness of school den- \\ tal screening: a cluster-randomized control trial. J Dent Res. 2006; \\ 924-8.
}

Design This was a cluster randomised controlled trial, in which the unit of randomisation was the school.

Intervention Three models of screening were tested against a control. A 'new' model of school dental screening incorporated a consensus view, from clinicians in the northwest of England, on a set of clinical criteria that would prompt a referral following a screening examination. A 'traditional' model involved the delivery of the existing school dental screening programme according to the principle that a child is referred if, in the opinion of the screening dentist, dental care is required. The third intervention tested was a dental information leaflet, distributed via the schools, which encouraged parents to examine their child's mouth and to take their child to a dentist if any problems were noted. Children a ttending the control schools received no intervention during the study period.

Outcome measure The main outcome measures were prevalence of teeth with active caries and mean number of teeth with active caries in the permanent or primary dentition. Secondary outcome measures were prevalence of oral sepsis, gross plaque or calculus, and dental trauma to incisor teeth.

Results Seventeen thousand and ninety-eight children in 169 clusters (schools) were eligible for inclusion in the study. One school was withdrawn from the study because of failure to agree to follow the trial protocol. Of the total, 15004 children were available for baseline examination in 168 schools and 13570 children received a baseline and outcome examination, representing $80.5 \%$ of the eligible population. After adjustment for clustering of children in schools, there was no significant difference in the reduction from baseline in untreated caries between the study groups in either the primary or permanent dentition. Similarly there were no significant differences across the four arms of the study in the secondary outcome measures of prevalence of sepsis, presence of gross plaque or calculus, and trauma to the permanent incisor teeth. In the traditional arms, $42 \%$ of children attended a dentist during the study period, with $41 \%$ in the new-model arm, $37 \%$ in the information-leaflet arm and $38 \%$ in the control arm. Although more children in the traditional and new-model arms of the study attended a dental appointment these differences were not statistically significant.

Conclusions School dental screening delivered according to three different models was not effective at reducing levels of active caries and increasing dental attendance in the population under study.

Address for correspondence: M Tickle, Oral Health Unit of the National Primary Care Research and Development Centre, School of Dentistry, University of Manchester, Higher Cambridge Street, Manchester M15 6FH, UK.

E-mail: martin.tickle@manchester.ac.uk

\section{Commentary}

The discussion about routine screening in schools for dental disease is both important and topical, since that screening is carried out extensively across the UK. Because screening requires co-operation from education departments and schools and is time-, personneland work-intensive, the question of efficacy is an interesting one.

As the authors note, the original aims of the UK school screening programme are a little vague; this paper aims to establish the impact of school dental screening on untreated dental disease and dental attendance at a population level. It reports on a cluster-randomised control trial which tested three models of screening against a control. A large population of over 13000 children aged 6-8 years took part in the study. The principal outcome measures were prevalence and mean number of teeth with active caries in both the permanent and primary dentitions, and attendance at a dentist in the 4-month period following the screening date.

The authors found no significant difference in the reduction from baseline in untreated caries between the study groups in either the primary or permanent dentitions. There was also no significant difference in the secondary outcome measures of prevalence of sepsis, presence of gross plaque or calculus, and trauma. Between the four groups, there was no significant difference in the number of children who attended a dentist in the 4-month period following the screening date. The authors did accept that 4 months could be argued as being too short a time period for dental treatment to have been completed and that the impact of the programme on a population will be diluted by nonattenders.

The study did not demonstrate that school dental screening is effective at reducing untreated dental caries or stimulating dental attendance at a population level. Although it could be argued that these two aims should be core to this screening programme, it could also be argued that screening is currently used to provide data at local level to establish disease levels in the local population in this age group and thus effectively plan and deliver local treatment and prevention services. Scotland currently has an extensive national dental inspection programme which is used to this effect (www.scottishdental.org/dentalinspection.htm).

Screening for dental disease is under consideration by the National Screening Committee (NSC) and an interim report that includes data from this study is available (URL www.library.nhs.uk/screening/viewResource. aspx? catID=10474\&resID=93689).

The conclusion of the authors is that, at present routine screening has no beneficial effect on [the pupils'] dental health. Before making a decision over whether to recommend cessation of the programme, however, they suggest that two questions should be addressed. First, could attendance resulting from screening be improved? Second, could treatment rates following referral be improved? They also ask what means might be used to maintain surveillance of dental health of children if the programme were to be abandoned. 
In terms of surveillance in England and Wales, the dental epidemiological surveys of 5-year- and 12-year-olds is undertaken biannually and co-ordinated by the British Association for the Study of Community Dentistry (BASCD); ${ }^{1}$ this may be an alternative but BASCD do not deliver the local-level information provided by the screening programme and this data collection is not without cost.

The NSC report indicated that it is those children from the more deprived areas who are less likely to attend a dental appointment following screening and this is likely be linked to parental attitudes and access to dental services ${ }^{2}$. Anecdotal reports suggest that the new Dental Contract in England and Wales may exacerbate access problems for individuals who have high disease levels. Although improving access to care is likely to improve attendance there is also an issue in relation to provision of treatment to children requiring care, as shown by the low care index (proportion of filled teeth relative to the number of decayed missing and filled teeth) seen in successive BASCD surveys.

It is important that screening programmes should be able to demonstrate that they are beneficial to the population and to those individuals identified as being at risk. The NSC is reviewing dental screening* but before a final decision is made the three questions posed in their interim report need to be answered.

\section{Jennifer Rodgers}

National Health Service (NHS) Fife, Leven, Fife and NHS Forth Valley, Stirling, Scotland, UK

1. Pine CM, Pitts NB, Nugent ZJ. British Association for the Study of Community Dentistry (BASCD) guidance on sampling for surveys of child dental health. A BASCD coordinated dental epidemiology programme quality standard. Community Dent Health $1997 ; 14: 10-17$

2. Tickle M, Milsom KM, Humphris GM, Blinkhorn AS. Parental attitudes to the care of the carious primary dentition. Br Dent J 2003; 195:451-455.

Evidence-Based Dentistry (2007) 8, 5-6. doi:10.1038/sj.ebd.6400460

* The NSC criteria for appraising the viability, effectiveness and appropriateness of a screening programme are reproduced on page 2 